Plasma Vertical Control with Internal and External Coils in Nest Step Tokamaks

by

C.E. Kessel, P. Heitzenroeder, and C. Jun

November 2000

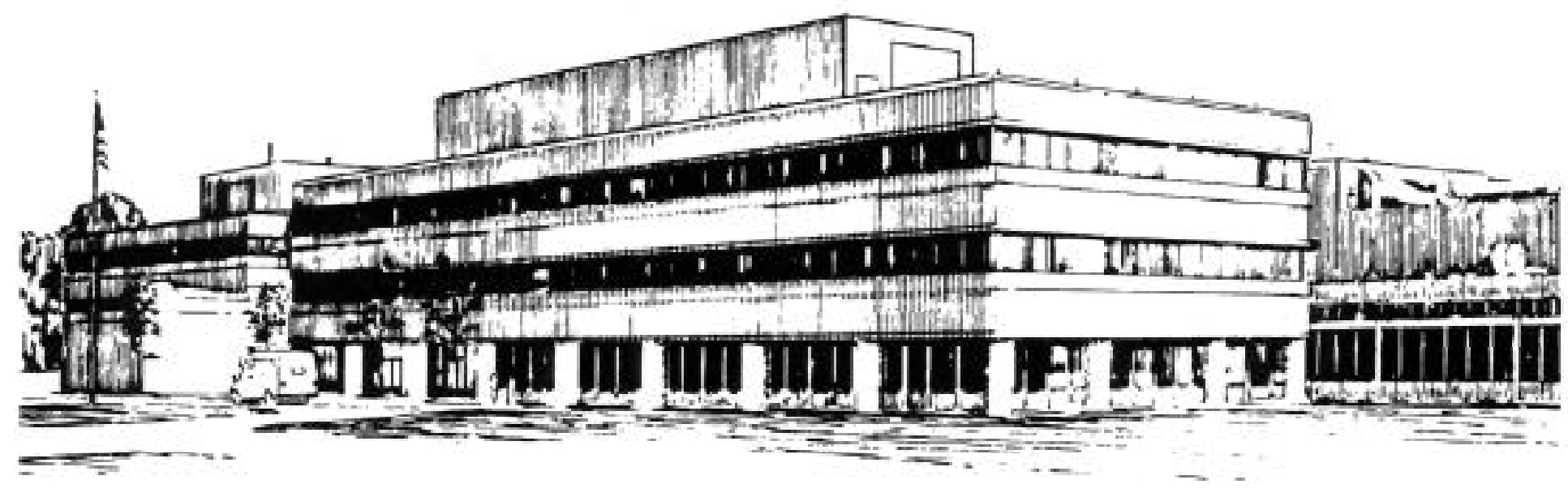




\section{PPPL Reports Disclaimer}

This report was prepared as an account of work sponsored by an agency of the United States Government. Neither the United States Government nor any agency thereof, nor any of their employees, makes any warranty, express or implied, or assumes any legal liability or responsibility for the accuracy, completeness, or usefulness of any information, apparatus, product, or process disclosed, or represents that its use would not infringe privately owned rights. Reference herein to any specific commercial product, process, or service by trade name, trademark, manufacturer, or otherwise, does not necessarily constitute or imply its endorsement, recommendation, or favoring by the United States Government or any agency thereof. The views and opinions of authors expressed herein do not necessarily state or reflect those of the United States Government or any agency thereof.

\section{Availability}

This report is posted on the U.S. Department of Energy's Princeton Plasma Physics Laboratory Publications and Reports web site in Calendar Year 2000. The home page for PPPL Reports and Publications is: http://www.pppl.gov/pub_report/

DOE and DOE Contractors can obtain copies of this report from:

U.S. Department of Energy

Office of Scientific and Technical Information

DOE Technical Information Services (DTIS)

P.O. Box 62

Oak Ridge, TN 37831

Telephone: (865) 576-8401

Fax: (865) 576-5728

Email: reports@adonis.osti.gov

This report is available to the general public from:

National Technical Information Service

U.S. Department of Commerce

5285 Port Royal Road

Springfield, VA 22161

Telephone: $1-800-553-6847$ or

(703) $605-6000$

Fax: (703) 321-8547

Internet: http://www.ntis.gov/ordering.htm 


\title{
Plasma Vertical Control with Internal and External Coils in Next Step Tokamaks
}

\author{
C. E. Kessel, P. Heitzenroeder, and C. Jun \\ ${ }^{1}$ Princeton Plasma Physics Laboratory, P.O. Box 451, Princeton, NJ 08543
}

\begin{abstract}
Vertical stability and control are examined for a tokamak configuration intended to be a generic representation of next step devices. Vertical stability calculations show that a critical resistive wall location can be determined for realistic structures, and that the introduction of small amounts of low resistivity material to an all steel structure can significantly reduce the vertical instability growth rate. Vertical control simulations show that internal control coils require significantly less feedback power than external coils, and that low resistivity materials can allow very low feedback powers or coils to be located externally with reasonable feedback powers.
\end{abstract}

\section{INTRODUCTION}

The vertical instability in elongated tokamaks is a critical design issue, and careful analysis is required to understand the tradeoff between increasing elongation and maintaining a reliable vertical control system. One of the cornerstones of advanced tokamaks has been to utilize plasma shaping to improve ideal MHD stability limits. However, it is well known that higher elongations lead to more vertically unstable plasmas, requiring more powerful feedback control systems. Most present tokamak experiments have poloidal field coils close to the plasma, and typically have thin metallic structures between the coils and the plasma. The next generation of burning and long pulse experiments will have poloidal coils farther from the plasma, and also more intervening structure between the coils and plasma. These features make the control of the vertical instability more difficult. The issue of whether feedback coils for plasma vertical position control should be placed close to the plasma (internal) or in the vicinity of the PF coils (external) has arisen many times. The TPX[1, 2] and KSTAR[3] design studies pursued coils located inside the vacuum vessel, while ITER EDA[4] design study pursued the use of poloidal field (PF) coils. However, these choices were often based on qualitative criteria. In order to examine the issue quantitatively, vertical stability and feedback control simulations are done. As it turns out, for a given plasma, the choice depends critically on the passive conducting structure which surrounds the plasma.

\section{TOKAMAK DESIGN USED IN ANALYSIS}

It is necessary to use a tokamak design that is sufficiently generic to provide more general guidance for future design efforts. The design used is referred to as the Advanced Tokamak Burning Experiment (ATBX)[5], which was an interim effort to reduce the cost of the ITER EDA final design, by reducing the device size and taking advantage of several technical extrapolations. The major parameters are; $\mathrm{I}_{p}=14.8 \mathrm{MA}, \mathrm{B}_{T}=6.35 \mathrm{~T}, \mathrm{R}=5.60 \mathrm{~m}, \mathrm{a}=1.75 \mathrm{~m}, \kappa_{x}=2.0$, and $\delta_{x}=0.7$. The critical features of the design which are important for plasma vertical stability and control are the plasma shape and the toroidally continuous structures. As in the ITER EDA the structures consist of a vacuum vessel (double-walled) and a backplate. However, the ATBX design has an up-down symmetric double null plasma. The plasma used in all these analyses has a $\beta_{p}$ of 0.1 and an $l_{i}$ of 0.8 , typical of start-of-flattop (SOF) plasmas which are usually the most unstable in the discharge. Since the plasma is double null, there are gaps in the structures at the top and bottom. The backplate structure is composed of steel that is $0.07 \mathrm{~m}$ thick, and the vacuum vessel is also steel with two shells each $0.035 \mathrm{~m}$ thick. The total toroidal resistance of 
the back plate is $68 \mu \Omega$, while the value for the vacuum vessel is $18.5 \mu \Omega$. Any contribution from first wall modules is neglected since we expect these to be made electrically discontinuous in the toroidal and poloidal directions (as was done in the ITER EDA design). A figure of the plasma, structure model, and the PF coils is given in Fig. 1. All analyses in the present work are axisymmetric.

\section{VERTICAL STABILITY ANALYSIS AND STRUCTURE DESIGN}

The passive conducting structure that surrounds the plasma plays a critical role in the plasma stability and control. The distance of the structure from the plasma, the structure thickness, perimeter in the poloidal plane, and resistivity, in conjunction with plasma parameters (elongation, $\beta_{p}$, and $l_{i}$ ) determine the growth time for the vertical instability. The growth time ultimately determines the power required in the feedback control coils. Another important measure of the vertical instability is the stability factor, which depends only on the structure's distance from the plasma boundary and it's poloidal coverage, and determines the margin to ideal MHD instability. This factor is defined as,

$$
f_{s}=1+\tau_{g} / \tau_{L / R}
$$

where $\tau_{g}$ is the vertical instability growth time, and $\tau_{L / R}$ is the longest lived up-down asymmetric eddy current mode in the surrounding structure. This is a measure of the inductive response and is independent of what the structural material is made of, because $\tau_{g} \propto \tau_{L / R}$. Both the stability factor and the vertical instability growth time are used in design. A series of vertical stability analyses were done with generic structure models to uncover the dominant effects of structure choices and to provide a small number of examples to pursue vertical control studies. The vertical stability analysis was done with Corsica[6], which solves a variational formulation of the $n=0$ stability and allows for a fully deformable plasma with resistive structures.

First we examined the vertical stability of plasmas with elongations of $2.2,2.0$, and 1.8 . The structure was modelled simply with outer and inner shells, both made of copper $0.025 \mathrm{~m}$ thick (with resistivity set to $\left.2.5 \times 10^{-8} \mathrm{ohm}-\mathrm{m}\right)$. An illustration of the shells used in the analysis is shown in Fig. 2, and cover the outboard and inboard sides of the plasma with gaps at the top and bottom for divertors. Shown in Fig. 3 is the plasma vertical growth rate (reciprocal of the growth time) as a function of the distance of the shells from the plasma boundary. It can be seen that these copper shells give growth rates of a few per second when the shells are close to the plasma, and increase significantly as the shells are moved away. The shell location where the growth rate rises rapidly is asymptoting to what is called the critical ideal wall location. At this location, if the shell is moved further away it will no longer have any influence in slowing the plasma motion, even if it were superconducting. However, at this location or any closer to the plasma, if the shell were superconducting, the plasma would be stable to the vertical instability. In terms of the stability factor, this location is where $f_{s}$ takes the value 1.0 , or where $\tau_{g} \ll \tau_{L / R}$, the growth time becomes Alfvenic and the plasma is considered uncontrollable.

Structures are actually resistive, so from a practical point of view this location is not useful. As demonstrated by the curves, if we located a copper shell (or any other metallic shell) near this location we would have very high growth rates and more significatly, the growth rate varies strongly for slight changes in the shell location (or plasma location relative to the shell). Instead we prefer to place the shells so that we have a low growth rate, and where the sensitivity to the precise location is weak. A new location, called the critical resistive wall location, is placed at the knee in the curves, prior to where they begin to rise rapidly. This location is a practical maximum distance for our shell, and corresponds to a stability factor of 1.2. It should be noted that a resistive shell can not stabilize the vertical instability, it can only slow it down. However, this slowing down is absolutely required for a realistic feedback control system to stabilize the instability. In addition, the curves indicate that lower elongations not only lead to lower growth rates, but that the shells can be placed further away from the plasma boundary. Had we 
used steel (with resistivity of 90.0 times $10-8 \mathrm{ohm}-\mathrm{m}$ ) for the shell structure, with its higher resistivity, the growth rates would be higher by the ratio of resistivities since $\tau_{g} \propto \tau_{L / R}$.

A significant improvement over single shells surrounding the plasma is to introduce another set of shells which work in conjunction with the original shells. The most effective arrangement for vertical stability is to locate the worse conductor (for example steel) closest to the plasma and the good conductor (for example copper) behind it. Fig. 4 shows two curves that are single shell models of steel $(0.07 \mathrm{~m}$ thick) and copper $(0.025 \mathrm{~m}$ thick $)$, and then two linear curves that are for fixed steel shells $(0.07 \mathrm{~m}$ thick, inboard and outboard) in combination with only an outboard copper shell $(0.025 \mathrm{~m}$ thick) or in combination with inboard and outboard copper shells $(0.025 \mathrm{~m}$ thick). For the linear curves, only the outboard copper shell is moved away from the plasma. These results indicate that adding a good conductor to an all steel structure can significantly lower the plasma vertical growth rate compared to an all steel structure. In the limit of the copper immediately behind the steel, the growth rate agrees with that from a single copper shell, for the inboard and outboard copper addition. For the outboard only copper addition, as the copper is moved further away the growth rate will approach the steel only case. For the steel and copper combination cases the fixed steel shell is located at 0.20 times the minor radius measured from the plasma boundary. The presence of the fixed steel shells close to the plasma make the growth rates increase more slowly as the copper is moved further away.

From a practical point of view, adding large copper shells whose only purpose is to slow down the plasma vertical instability is not realistic. The steel shells are necessary for structural and vacuum reasons, however, the copper should be minimized as much as possible. To reduce the copper shells, the copper is eliminated in sections beginning from the midplane and moving upward (and downward) both on the inboard and outboard. Fig. 5 illustrates the copper shell reductions, and the steel structures are fixed as in the previous analysis. The copper can be removed from the midplane area as much as $\pm 25^{\circ}$ with virtually no impact on the instability growth rate. This is due to the eddy current distribution induced in the shells when the plasma moves vertically, which are very small near the midplane both on the inboard and outboard, and so do not contribute to stabilization. Shown in Fig. 6 is the growth rate as a function of the outboard copper distance to the plasma, for the case above of fixed steel structures and fixed inboard copper shell. The additional points show how the growth rate varies as more copper is removed from both the inboard and outboard copper shells. Only two outboard copper shell locations are shown. The physical criteria that typically determines how much of the copper to remove are the midplane port clearances and the divertor. For the present case we will remove the outboard copper structure from the midplane over the range of $\pm 65^{\circ}$. The inboard copper is then made to have the same vertical extent as this outboard shell, which for the given ATBX design is $2.55 \mathrm{~m}$ to $3.75 \mathrm{~m}$. The inboard copper is particularly useful for low pressure plasmas and radially inward shifted plasmas. This final copper and steel structure is shown in Fig. 5 denoted by $65^{\circ}$.

Based on the above analysis two structure models are chosen, an all steel structure composed of the steel backplate and vacuum vessel, and the same structure augmented with $0.025 \mathrm{~m}$ thick copper plates between heights of $2.55 \mathrm{~m}$ and $3.75 \mathrm{~m}$, located behind the backplate on the outboard side and in front of the inboard vaccum vessel. The backplate is a distance of 0.25 times the minor radius from the plasma boundary, and is only on the outboard side, since the inboard side vacuum vessel is already close to the plasma and can serve as the structural member there. The structure models to be used in the vertical control calculations are shown in Fig. 1. The resulting vertical instability growth times for these two structures are $56 \mathrm{~ms}$ and $280 \mathrm{~ms}$, respectively. The structure L/R times are $200 \mathrm{~ms}$ and $780 \mathrm{~ms}$, the stability factors are 1.28 and 1.36 , and the total toroidal resistances are 14.5 and $4.3 \mu \Omega$, respectively.

Since there exists considerable vertical stability and structural design analysis for the ITER EDA it serves as a useful benchmark for the present studies.. The plasma elongation for ITER EDA is 1.75 (average of upper and lower elongations), and is a single null. The distance of the backplate (the closest toroidally continuous conductor) from the plasma boundary is 0.22 times the minor radius. The range of 
growth rates for the vertical instability is 1.0-1.8 per second depending on the point in the discharge. For the reference discharge, the X-point formation (XPF) and the start of flattop (SOF) plasmas are the most unstable since they are at or near full shape, but at low pressure and higher $l_{i}$. The backplate is made of steel $0.12 \mathrm{~m}$ thick, with a total toroidal resistance of $12.5 \mu \Omega$. In addition, the backplate is both toroidally and poloidally continuous except for the lower divertor opening. Outside of that, the vacuum vessel, also toroidally and poloidally continuous, is made of steel with a varying thickness between 0.08 and 0.12 $\mathrm{m}$, with a total toroidal resistance of $8.5 \mu \Omega$. The plasma vertical position control is accomplished with the PF coils located outside the vacuum vessel and is integrated as part of the plasma shape control. Considerable efforts have been put into reducing the power used in controlling the plasma position and shape during disturbances. Modern optimal control algorithms have been developed for this device[7].

The growth rates in the ITER EDA design are smaller than those for the ATBX design, even though only steel is used. This observation can be traced back to a number of features; 1) ITER has a lower elongation, 2) the backplate is thicker, 3) the vacuum vessel is thicker, and 4) the larger device has lower toroidal resistance due to larger poloidal perimeters for the backplate and vacuum vessel. Each of these introduce a factor, which when combined explains the difference quite accurately.

\section{VERTICAL POSITION FEEDBACK CONTROL ANALYSIS}

It is of interest to understand the tradeoff between the two ends of the spectrum, using internal control coils with large growth rate plasmas (all steel structures) and using external control coils with small growth rate plasmas (copper added to steel structures). In order to examine this the ATBX structure model is used with vertical position feedback coils located in three possible locations, all on the outboard side; 1 ) in front of the backplate (behind any modules or tiles that would serve as the first wall), 2) just behind the backplate, and 3) just behind the vacuum vessel. These locations are all at the same poloidal angle measured from the geometric center of the plasma so that the radial field created is only a factor of minor radial distance. These locations are shown in Fig. 1. It should be noted that although studies have shown feedback coils located on the inboard side, near the midplane, are very effective for vertical position control[8], the practical application of this is difficult. The inboard radial build of next step devices strongly affects their cost, and the integration of vertical control into the inboard solenoid coils is challenging. Also, a recent examination of internal and external coils for vertical control has been done on the TCV experiment[9].

The vertical position control simulations are done with TSC[10] (Tokamak Simulation Code), with three specific disturbances; 1) a $0.05 \mathrm{~m}$ step response, 2) $0.02 \mathrm{~m}$ RMS random disturbance, and 3) 0.05 $\mathrm{m}$ drift and recover. Since control results are sensitive to the gains chosen, a fixed prescription for the gains was used. The control uses proportional-derivative feedback on the poloidal flux difference through sensors located equally above and below the midplane on the inboard side. The flux sensor locations are chosen to intercept the largest asymmetric flux contour that touches the inboard vacuum vessel, making them as sensitive as possible. This is shown in Fig. 7, where the asymmetric flux contours are given when the plasma is about $0.01 \mathrm{~m}$ off the midplane. The gains are chosen to reduce the feedback control power. The proportional gain is taken to be 1.25 times the minimum stable gain. The minimum stable gain is derived from the product of the gain and the difference between the mutual inductances between the control coils and the flux sensors, and it must be -1.0 or less (more negative) to obtain stable control.

$$
g_{p}\left(M_{c 1, s 1}+M_{c 2, s 2}-M_{c 1, s 2}-M_{c 2, s 1}\right) \leq-1.0
$$

where $g_{p}$ is the proportional gain, and $M_{c, s}$ are mutual inductances between coils and sensors. This can be understood by examining the poloidal flux created at the sensor locations by the feedback control coils.

$$
\psi_{s 1}=M_{c 1, s 1} I_{c 1}+M_{c 2, s 1} I_{c 2}
$$




$$
\psi_{s 2}=M_{c 1, s 2} I_{c 1}+M_{c 2, s 2} I_{c 2}
$$

where $\psi_{s 1}$ and $\psi_{s 2}$ are poloidal fluxes, and $I_{c 1}$ and $I_{c 2}$ are coil currents. The feedback law uses current control defined by,

$$
I_{\text {feedback }}=g_{p}\left(\psi_{s 1}-\psi_{s 2}\right)+g_{d}\left(\dot{\psi_{s 1}}-\dot{\psi_{s 2}}\right)
$$

where $g_{d}$ is the derivative gain. Recall that the coils have current flowing in opposite directions to each other. Considering only the proportional term, the feedback current must be sufficient to, at a minimum, cancel the existing flux difference. Substituting eqns. 3 into eqn. 4, the stability criteria can be recovered. The derivative gain is then chosen to provide a critically damped response to a step request. This approach is used to find the gains for each structure/coil location combination. All of the disturbances are simulated by flux offsets through the sensor signals in the feedback loop. So the actual feedback law used in the simulations is,

$$
I_{f e e d b a c k}=g_{p}\left(\psi_{s 1}-\psi_{s 2}-\Delta \psi_{o f f}\right)+g_{d}\left(\dot{\psi_{s 1}}-\dot{\psi_{s 2}}-\Delta \dot{\psi_{o f f}}\right)
$$

where $\Delta \psi_{\text {off }}$ is the offset in flux. Although the other disturbances are straight forward, for the random disturbance this offset is prescribed by,

$$
\Delta \psi_{\text {random }}=\Delta \psi_{\circ} \sqrt{\frac{\tau}{T}} \sum_{n=1}^{\infty} \exp \left(-\frac{\omega_{n}^{2} \tau^{2}}{8}\right) \cos \left(\omega_{n} t-\phi_{n}\right)
$$

Here $\Delta \psi_{0}$ is a flux magnitude, $\tau$ is the autocorrelation time set equal to the vertical growth time, $T$ is the period, $\omega_{n}=2 \pi n / T$, and $\phi_{n}$ is a uniform random phase $\left(-\pi \leq \phi_{n} \leq \pi\right)$. The voltage for the feedback coil is provided by a proportional law given by

$$
V_{\text {feedback }}=g_{v}\left(I_{\text {feedback }}-I\right)
$$

where the $g_{v}$ is a proportional gain, $I_{\text {feedback }}$ is given by eqn. 3, and $I$ is the present value of the coil current. The value of $g_{v}$ is set at a value of 0.002 for all cases. The control is insensitive to the precise value of this gain. A voltage maximum is applied, which is determined by the lowest voltage maximum that can be tolerated among the 3 disturbances, and is rounded up to the nearest 5 volts. It turns out that although the step response and drift and recover disturbances can tolerate lower voltage maximums, the random disturbance sets this limit in all cases, due to the continuous short timescale oscillations.

Shown in Figs. 8 through 10 are examples of the three disturbance simulations for the coils located inside the backplate, and for steel only structure. In this case the maximum current and voltage is found to be $165 \mathrm{kA}$-turns and $150 \mathrm{~V} / \mathrm{turn}$. Shown in Table 1 are the maximum current, voltage, and power (MVA) for the feedback control coils for the various cases examined. It was found that the drift and recover and random disturbances had very similar current (and power) requirements, while the step response requirements were typically half or less. For the steel only structure model, the feedback control powers are high, even when the coils are located very close to the plasma. With the addition of copper the feedback control powers are significantly less. In fact, the feedback powers between the steel only and steel with copper scale roughly with the vertical instability growth times, $56 \mathrm{~ms}$ and $280 \mathrm{~ms}$, respectively. It should be noted that the currents and voltages (and consequently the powers) are functions of the magnitude of the disturbances, so that smaller disturbances would reduce the values shown, but the relative behavior would be preserved. To demonstrate this the case with steel and copper structure and the feedback coil located behind the vacuum vessel was simulated for $0.012 \mathrm{~m}$ RMS and $0.035 \mathrm{~m}$ RMS random disturbances. The results in Table 2 show that the peak power scales roughly linearly with the disturbance magnitude.

\section{DISCUSSION}


A generic study of vertical stability and control is presented, using a specific gain prescription, radially increasing coil locations, axisymmetric structure models, and a PD feedback control algorithm. The vertical control simulations indicate that internal control coils require significantly less power than external control coils. In addition, the use of a copper addition to an all steel structure combined with feedback coils behind the vacuum vessel (the farthest location from the plasma) requires lower feedback power than an all steel structure combined with feedback coils located in front of the backplate. It is clear that the design of a vertical position feedback system also involves the design of the conducting structures. Introduction of low resistivity materials (i.e. copper, aluminum, tungsten) to augment existing structural materials (i.e. steel) can reduce vertical instability growth rates allowing lower feedback power or feedback coils to be placed further from the plasma.

The ITER EDA design obtained low growth rates by a combination of low elongation, thick steel structures, and large poloidal perimeters for these structures (resulting in low toroidal resistance). In addition, optimal control strategies were used to spread the feedback control among all the PF coils and reduce the power required. The plasma elongation is restricted by this approach to avoid excessive feedback powers. In recent power plant designs[11], the PF coils are very far from the plasma due to maintenance requirements, so that the vertical position feedback coils are located as close as possible to the plasma behind the neutron shield (which is roughly equivalent to behind the vacuum vessel for the present study). The structures present in the blanket and shield are typically not adequate to slow the vertical instability, so tungsten (due to its low resistivity and high melting temperature) conducting structures are located as close as possible to the plasma to provide for plasma elongations in the range of 1.9-2.2 and a sufficiently low feedback control power. This makes the "reactor relevance" of using the large superconducting PF coils for vertical position control questionable, and the use of dedicated internal coils with low resistivity materials the more relevant direction.

As the PF coils move further away from the plasma, and more complex intervening structures are required in next step tokamaks, the design for vertical stability will change from the present experimental situation of nearby PF coils and thin metallic vacuum vessels. Since these devices will require very accurate vertical control due to large plasma heat fluxes and more stringent first wall and divertor tolerances, the use of an internal coil vertical control approach, particularly in combination with some low resistivity material, appears a superior approach based on feedback power.

\section{Acknowledgement}

Work supported by U.S. DOE Contract DE-AC02-76-CHO3073

\section{References}

[1] Davidson, R. C., Goldston, R. J., Neilson, G. H., et al., Phys. Plasmas, 2 (1995) 2417.

[2] Kessel, C. E., Jardin, S. C., Neilson, G. H., "Plasma Vertical Stability and Feedback Control for TPX", Proc. 15th Symp. Fusion Engineering, IEEE 93CH3348-0, (1994) 638.

[3] Lee, G. S., Kim, J., Hwang, S. M., et al., Nucl. Fusion, 40 (2000) 575.

[4] Wesley, J., Bartels, H.-W., Boucher, D., et al., Nucl. Fusion, 40 (2000) 485.

[5] Porkolab, M., “Optimized Pulsed Burning Plasma Experiment in a Superconducting Tokamak Facility", 17th IAEA Fusion Energy Conference, F1-CN-69/FTP/13, Yokohama, Japan, October, 1998.

[6] S. W. Haney, L. D. Pearlstein, R. H. Bulmer, and J. P. Freidberg, Plasma Physics Reports, 23 (1997) 789. 
[7] Portone, A., Albanese, R., Gribov, Y., et al., Fus. Tech., 32 (1997) 374.

[8] Lister, J. B., Martin, Y., Moret, J.-M., Nucl. Fusion, 36 (1996) 1547.

[9] Hofmann, F., Dutch, M. J., Favre, A., et al., Nucl. Fusion, 38 (1998) 399.

[10] S. C. Jardin, N. Pomphrey, and J. Delucia, J. Comput. Phys., 66 (1986) 481.

[11] Najmabadi, F., and the ARIES Team, Fus. Engr. and Design, 38 (1997) 1. 
Table 1: Feedback Control Currents, Voltages, and Powers

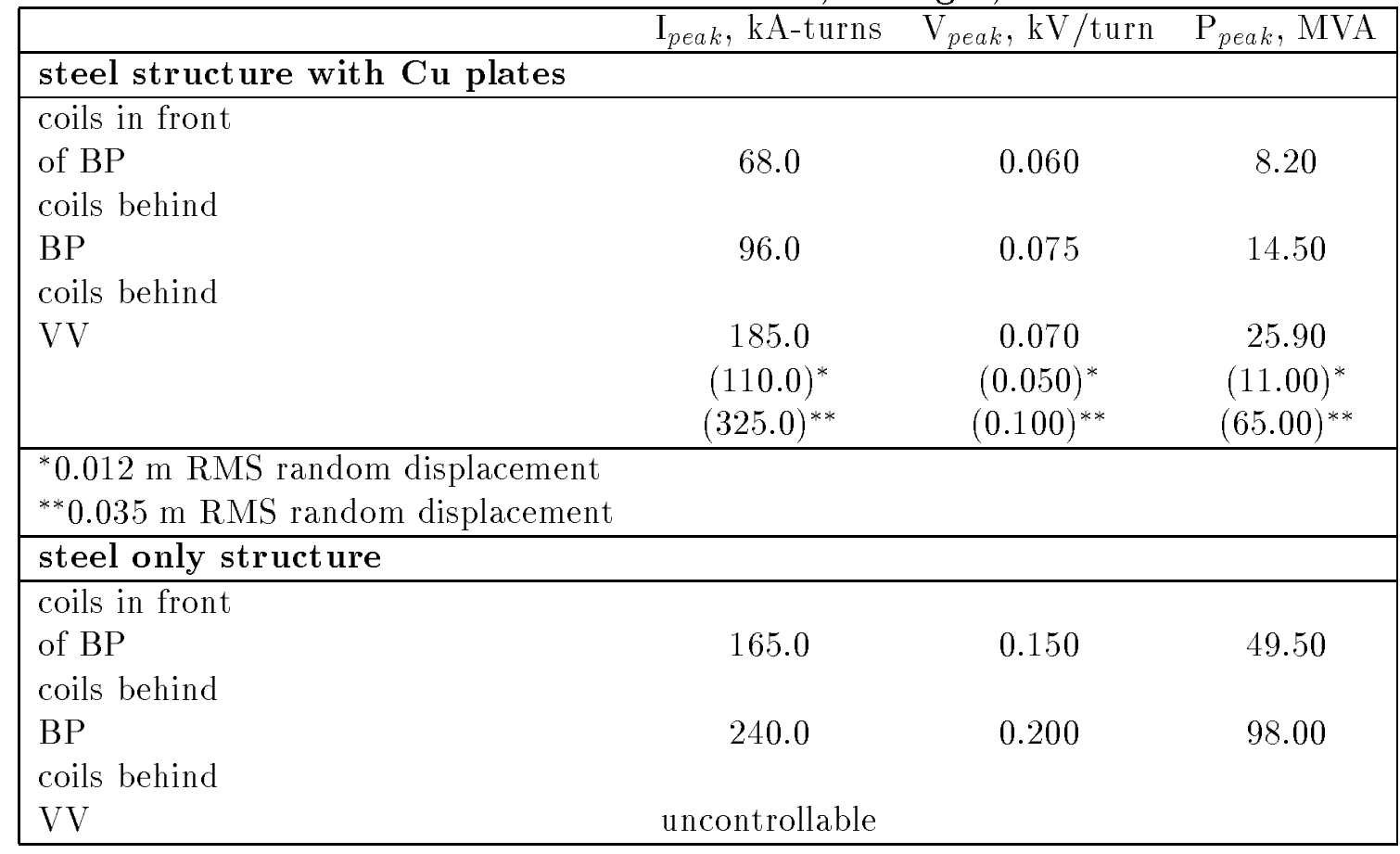




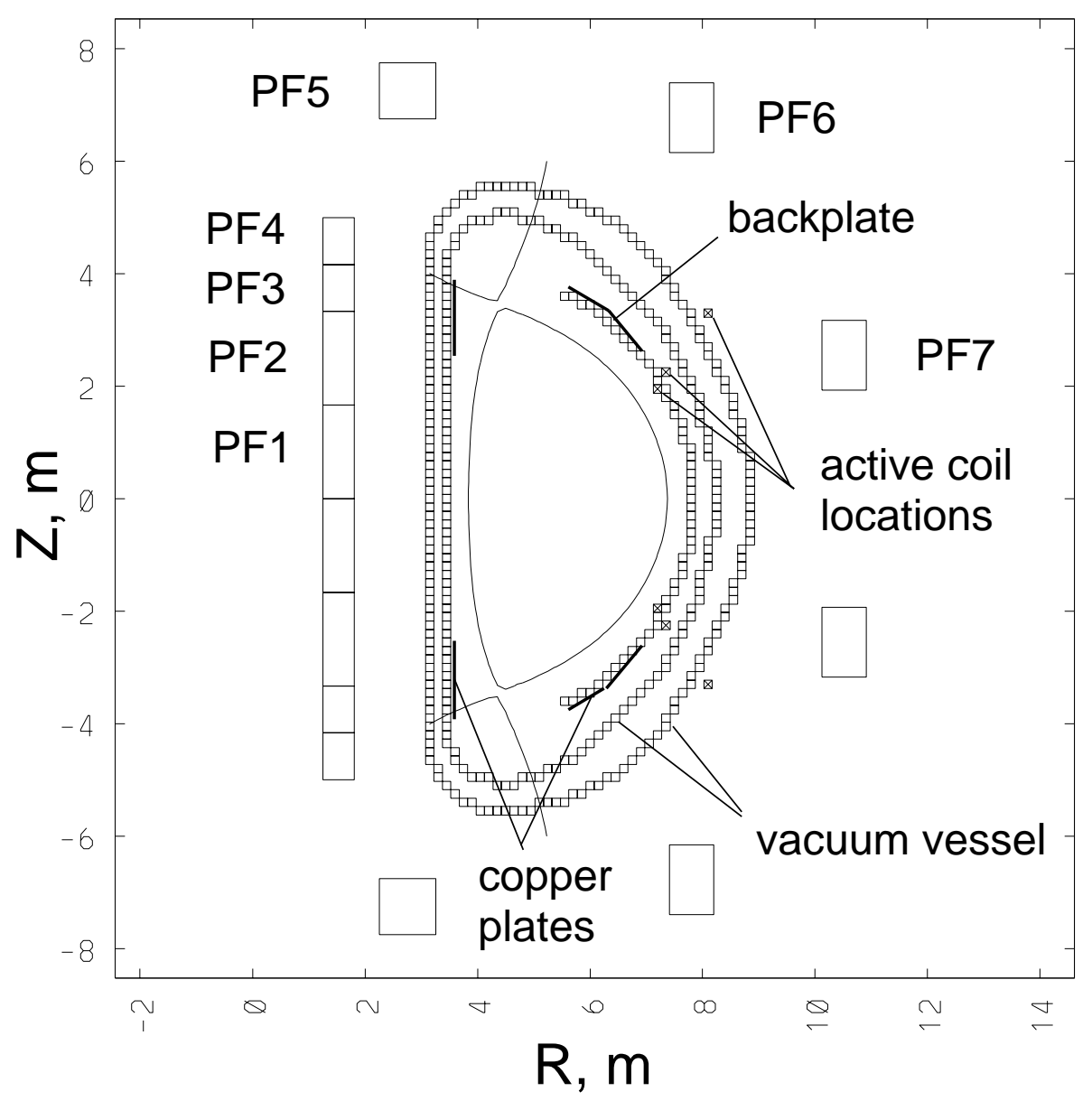

Figure 1: Model of plasma, structures, and PF coils for the ATBX design. 


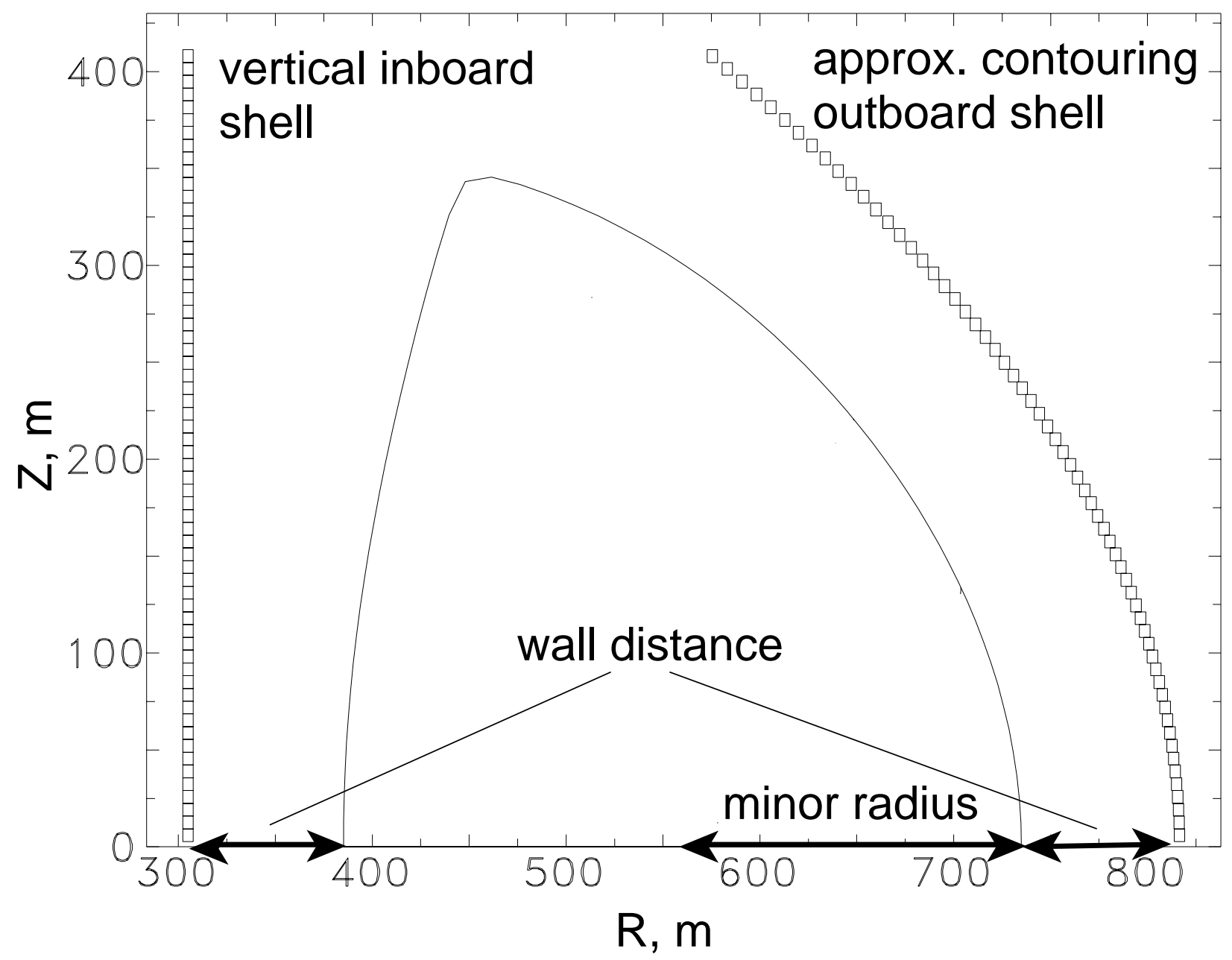

1

Figure 2: Illustration of the inboard and outboard shells used in the vertical stability analysis. 


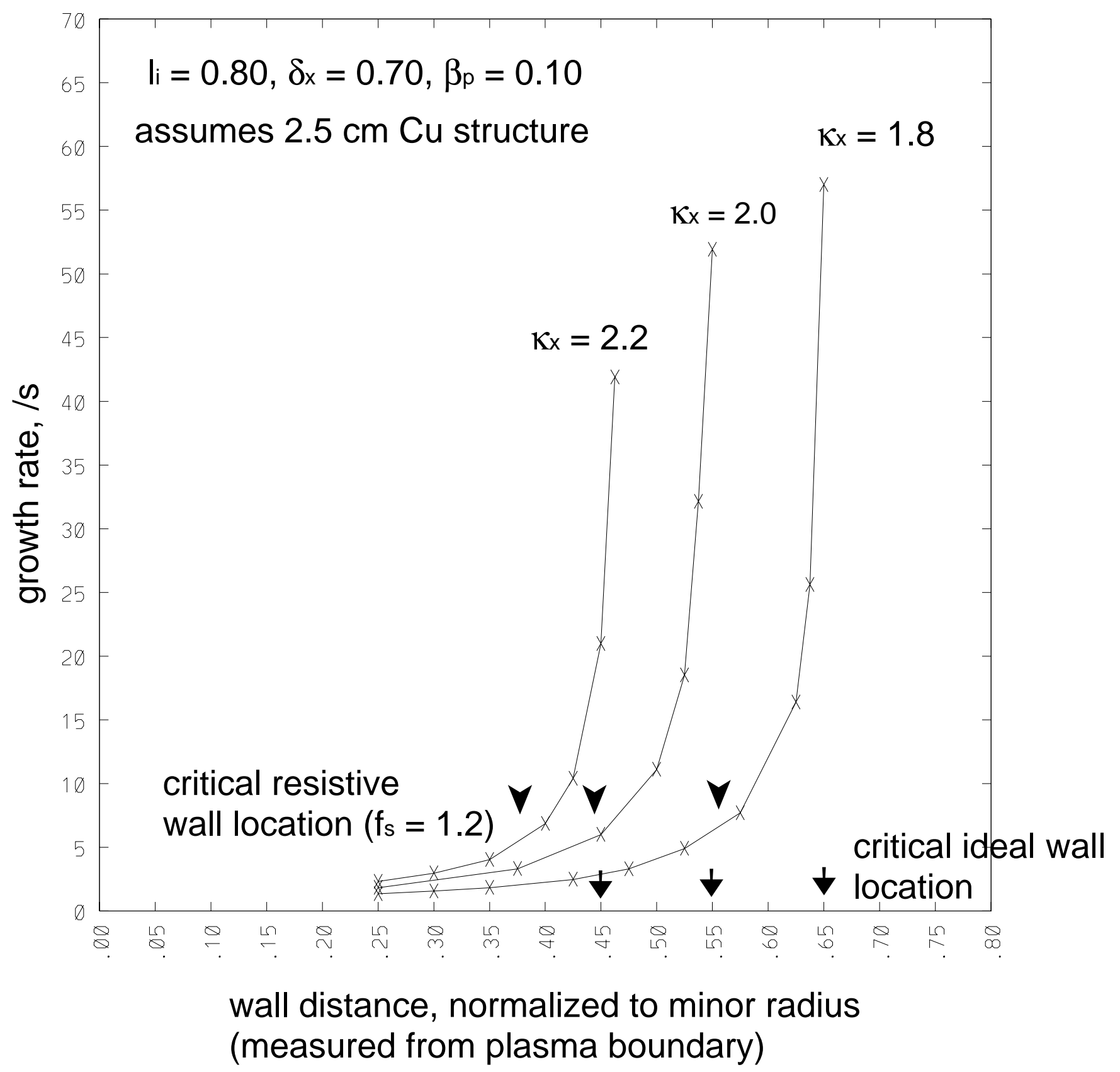

Figure 3: Plasma vertical instability growth rate as a function of the shell distance from the plasma boundary, normalized to the minor radius, for elongations of $1.8,2.0$, and 2.2 . 


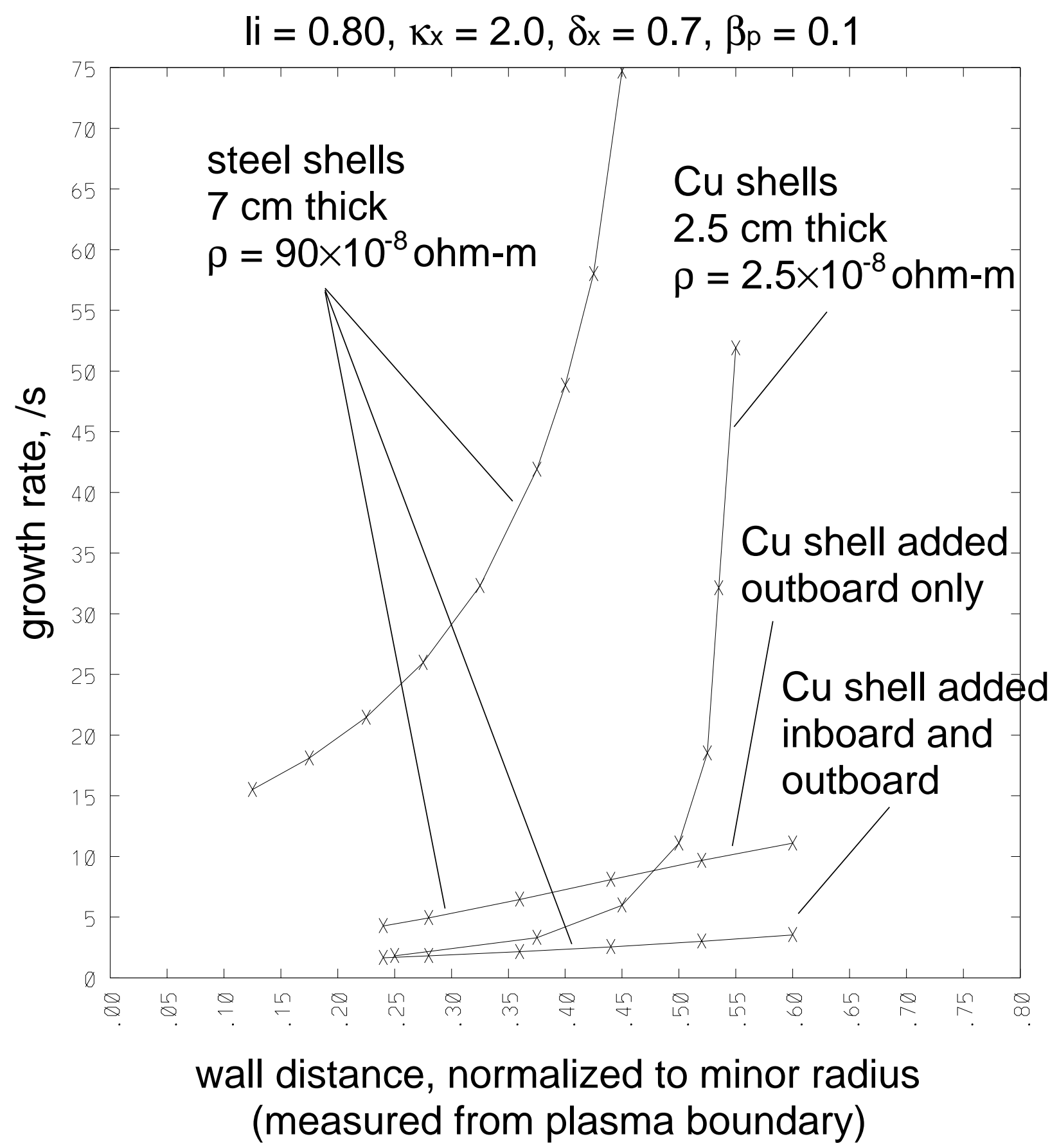

Figure 4: Plasma vertical instability growth rate as a function of the shell distance from the plasma boundary, normalized to the minor radius, for all steel inboard and outboard shells, all copper inboard and outboard shells, steel inboard and outboard shells with a copper shell added on the outboard only, and steel inboard and outboard shells with copper inboard and outboard shells added. 


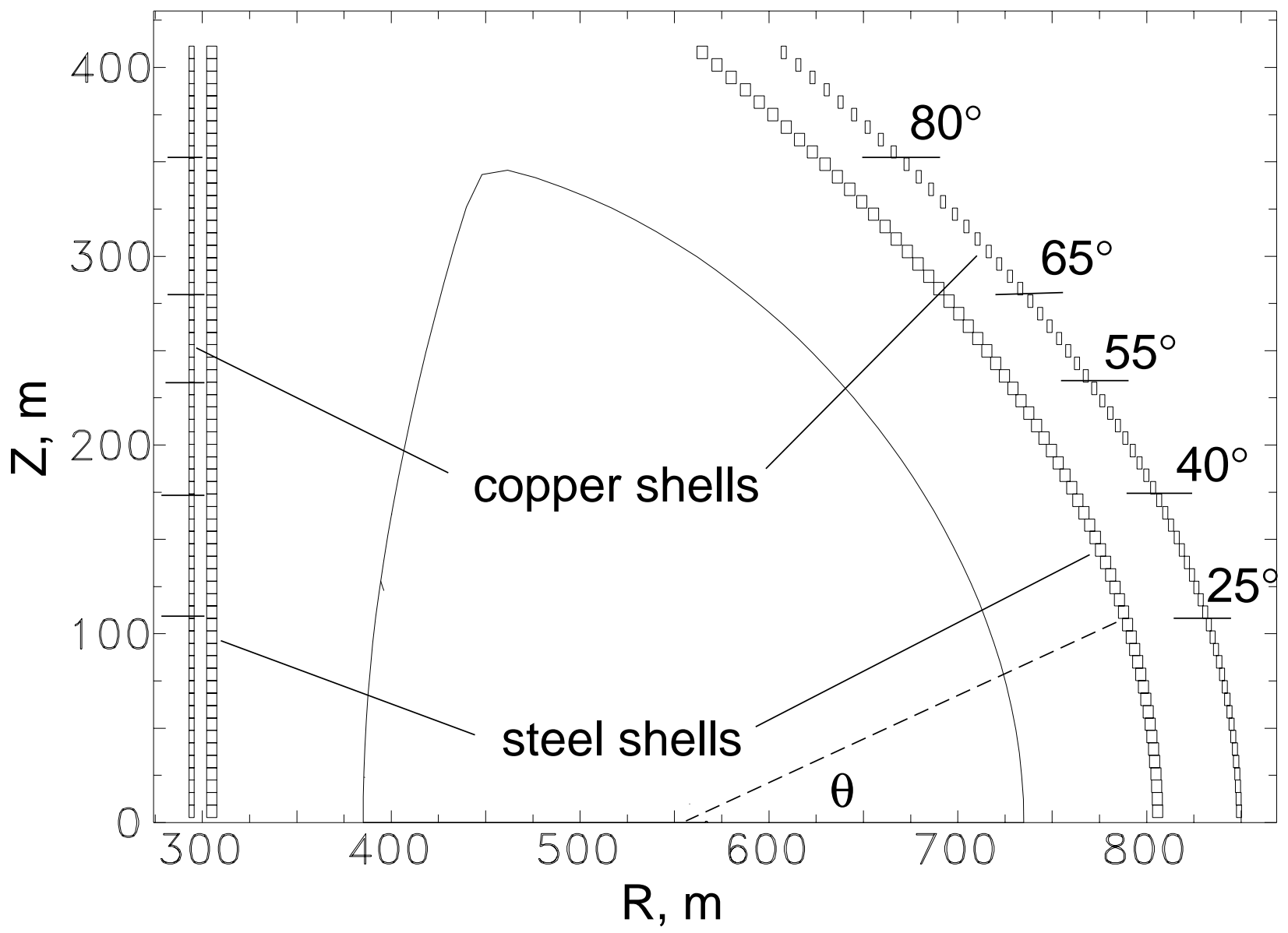

Figure 5: Illustration of the steel and copper combination configuration for the vertical stability analysis, showing how the copper shell is reduced. 


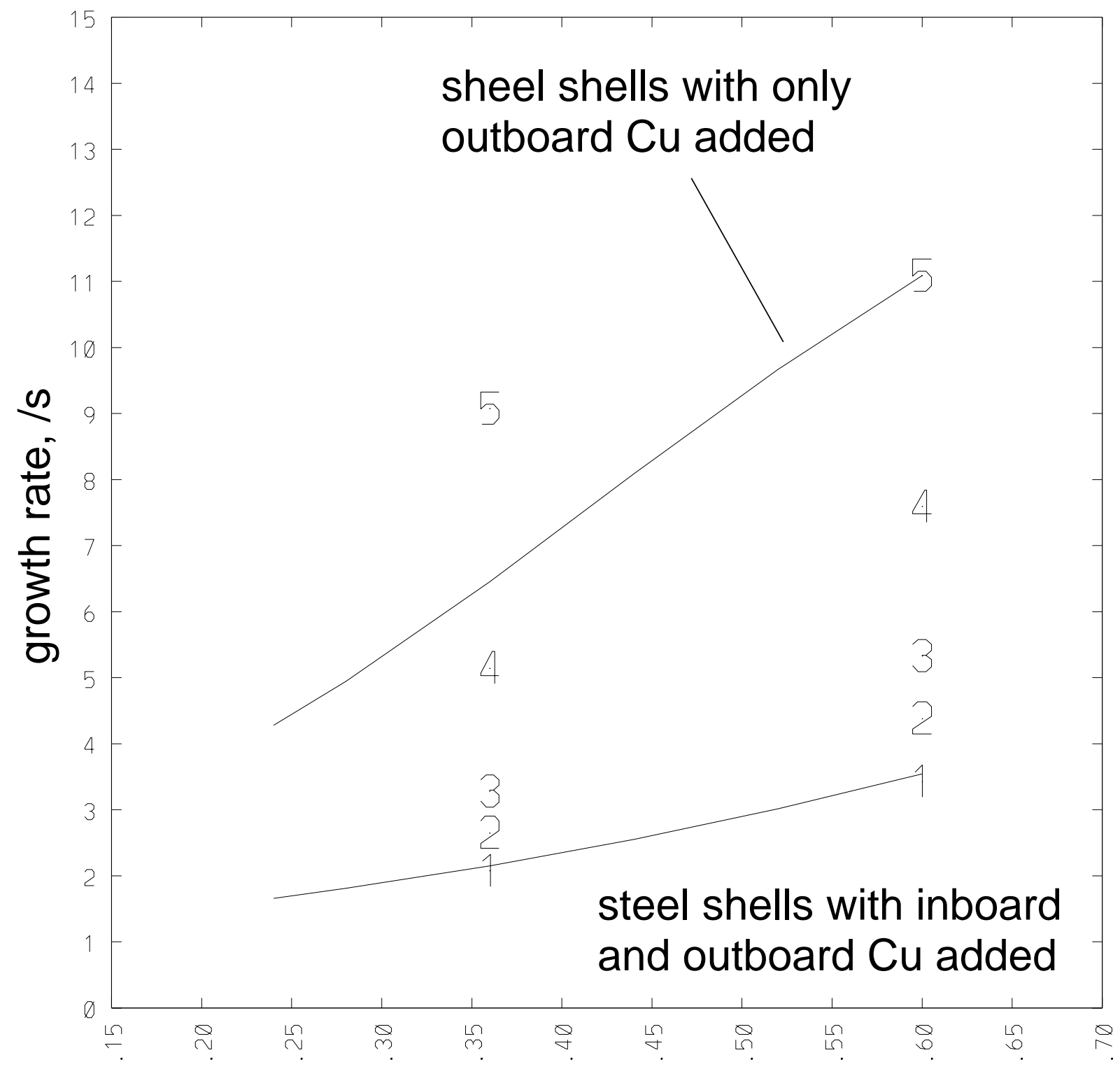

\section{wall distance, normalized to minor radius (measured from plasma boundary)}

Figure 6: Plasma vertical instability growth time as a function of the shell distance from the plasma boundary, normalized to the minor radius, for fixed inboard and outboard steel shells with copper added both inboard and outboard, while the copper coverage is reduced; 1) $25^{\circ}$, 2) $40^{\circ}$, 3) $55^{\circ}$, 4) $65^{\circ}$, and 5) $80^{\circ}$. The curves are the same as those shown in Fig. 4. 


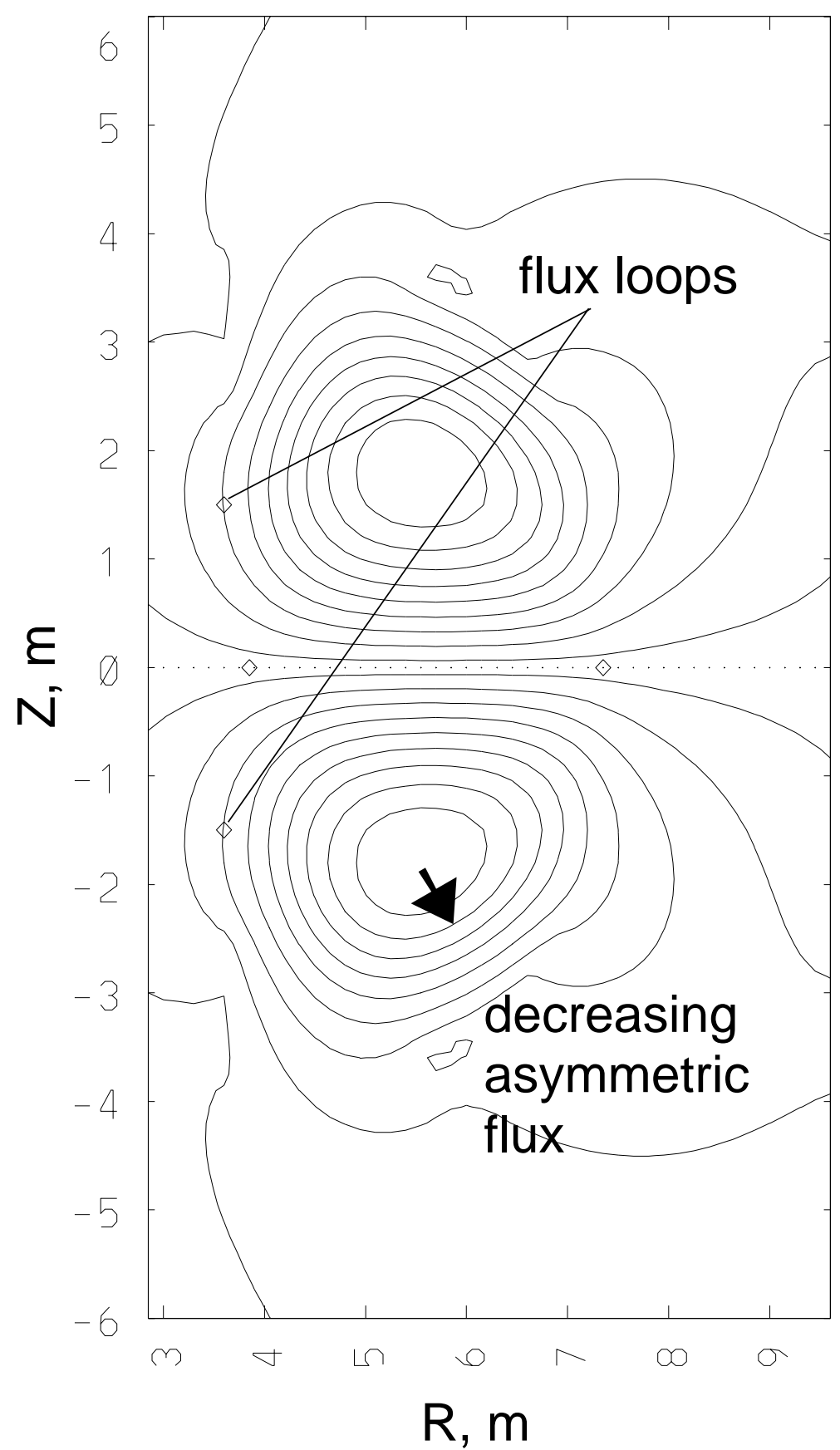

Figure 7: Plot of asymmetric flux contours when the plasma is $0.01 \mathrm{~m}$ off the midplane and the flux sensor locations on the inboard wall which are chosen to contact the contour with the largest value. The maximum asymmetric flux occurs at the center of the smallest contour inside the plasma, and decreases as one moves outward. 


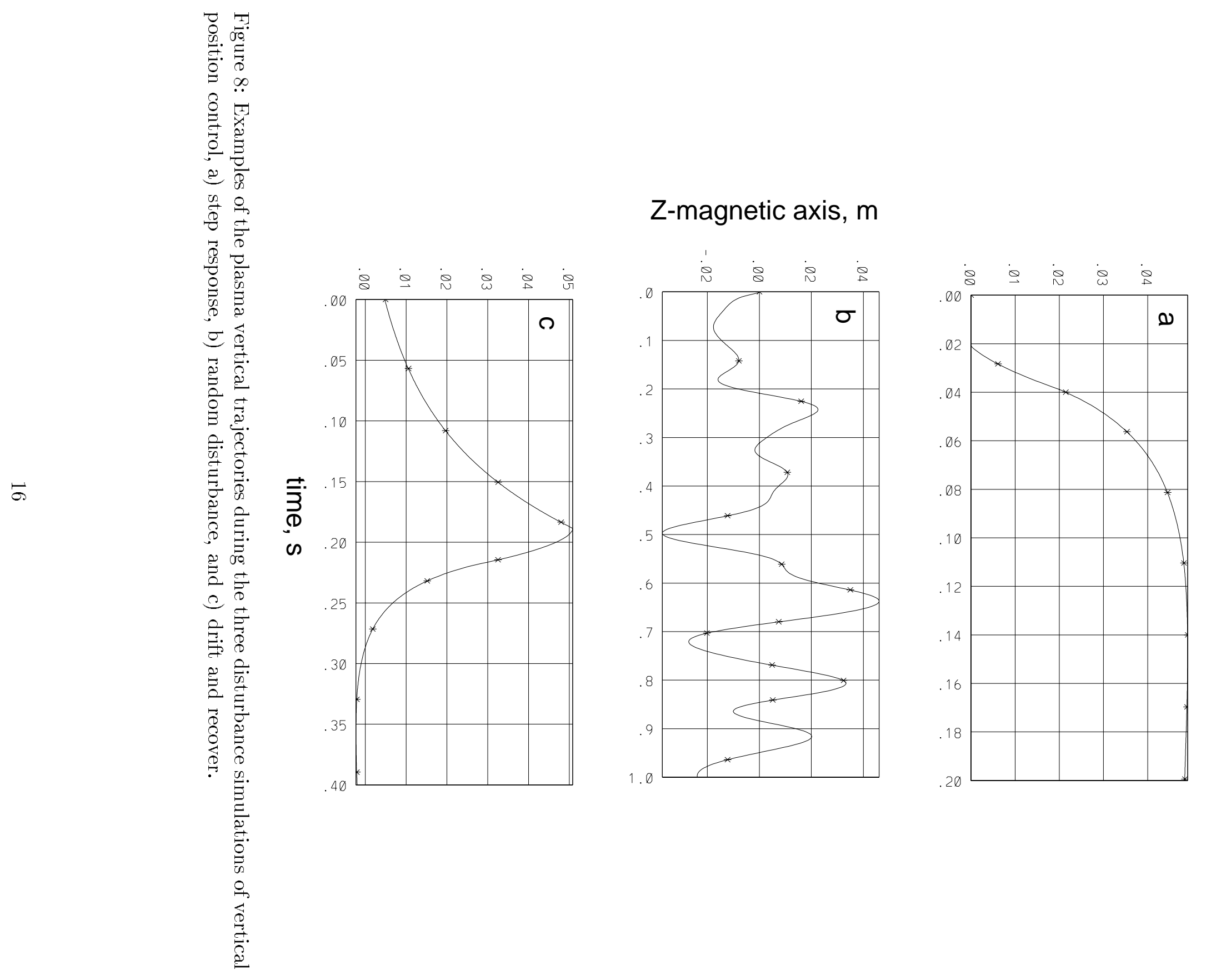




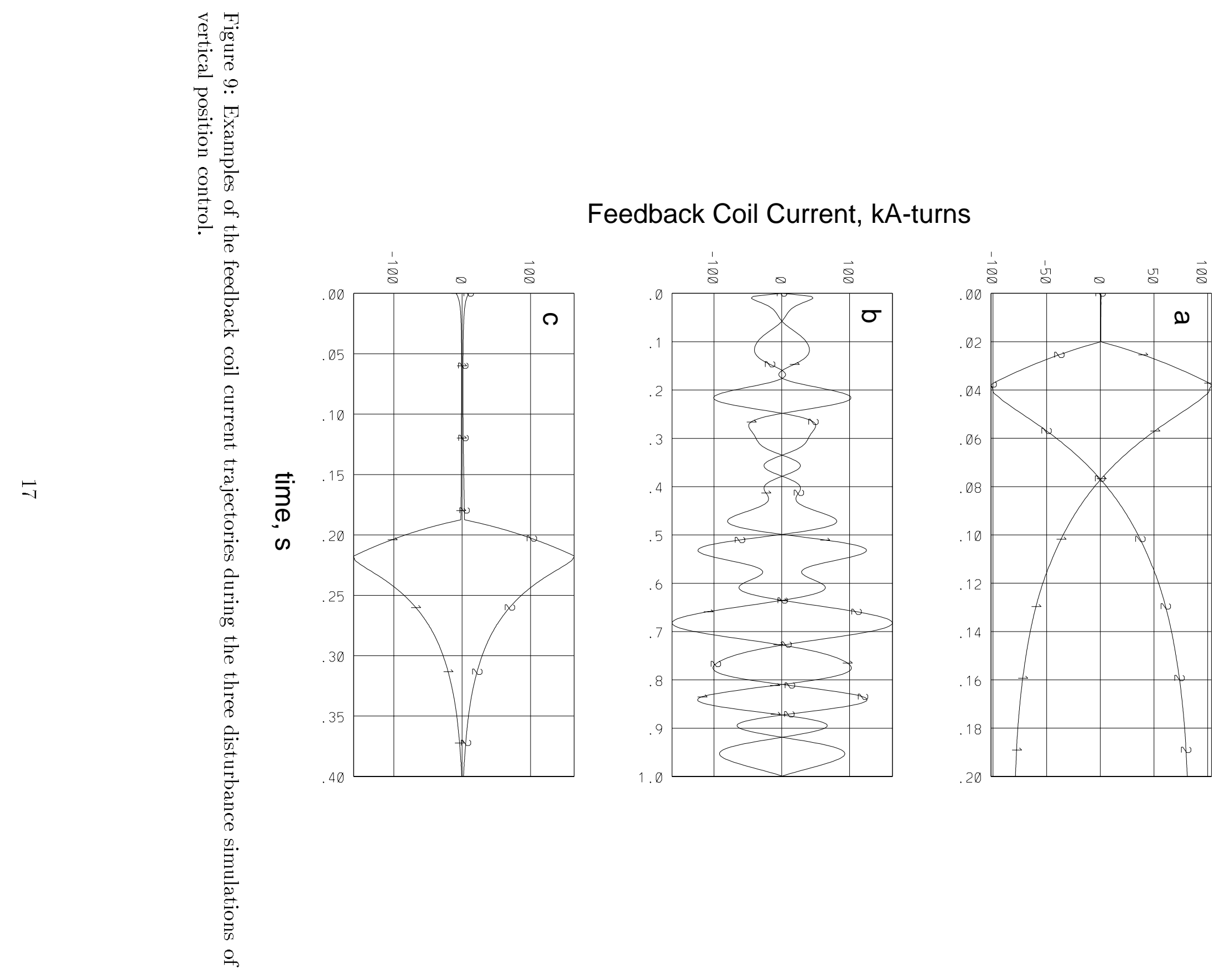




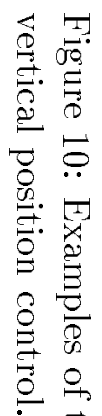

0

㕝

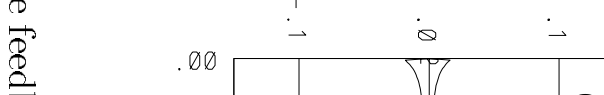

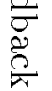

8.

Ј

$\frac{0}{\frac{1}{7}}$

0

声泀.

要

䒠

要

孛

$\stackrel{\overrightarrow{5}}{\vec{\Xi}}$

$\overrightarrow{5}$

क्.

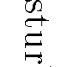

$\stackrel{\vec{Q}}{\vec{g}}$

อ

$\stackrel{\square}{3}$.

F

$\stackrel{\overrightarrow{0}}{\vec{g}}$

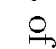

\section{Feedback Coil Voltage, kV/turn}
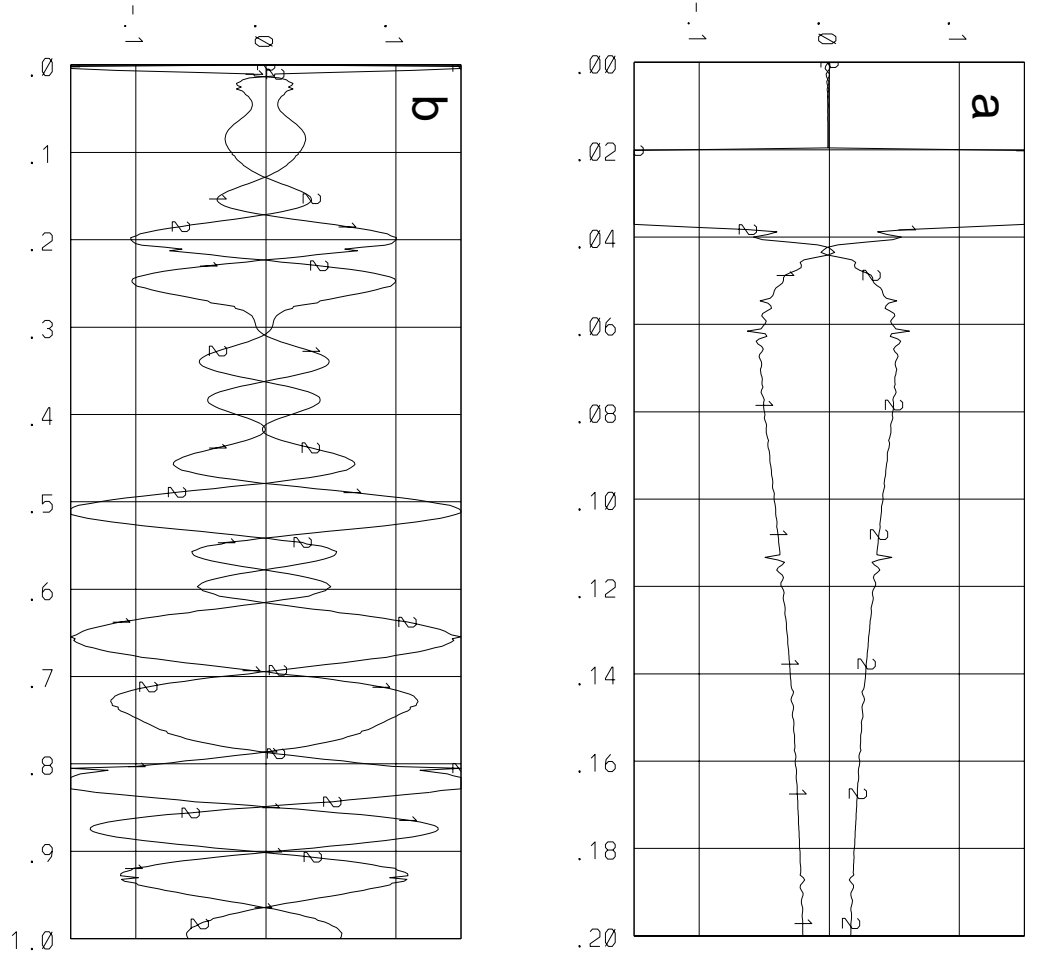
The Princeton Plasma Physics Laboratory is operated by Princeton University under contract with the U.S. Department of Energy.

\author{
Information Services \\ Princeton Plasma Physics Laboratory \\ P.O. Box 451 \\ Princeton, NJ 08543
}

Phone: 609-243-2750

Fax: 609-243-2751

e-mail: pppl_info@pppl.gov

Internet Address: http://www.pppl.gov 